\title{
Malignant Bone Marrow Neoplasm
}

National Cancer Institute

\section{Source}

National Cancer Institute. Malignant Bone Marrow Neoplasm. NCI Thesaurus. Code C35501.

Malignant neoplasms that either originate from the bone marrow (e.g. myeloid leukemias) or involve the bone marrow as secondary-metastatic tumors (e.g. metastatic carcinomas to the bone marrow). --2003 